\title{
ANÁLISIS DEL PERFIL SOCIODEMOGRÁFICO DE LAS ADOLESCENTES EMBARAZADAS ASISTIDAS EN UNA UNIDAD DE ATENCIÓN INTEGRAL DE UN HOSPITAL DE SANTO DOMINGO, REPÚBLICA DOMINICANA, PERÍODO FEBRERO-ABRIL, 2017
}

\section{Analysis of the sociodemographic profile of pregnant adolescents assisted in a comprehensive care unit of a hospital in Santo Domingo, Dominican Republic, February-April, 2017}

\author{
Omar de la Rosa*, Luis J. Guzmán, ${ }^{* *}$ Melysa I. Martínez** , Paulette P. Reyes**
}

\author{
DOI: http://dx.doi.org/10.22206/cysa.2018.v2i1.pp41-47
}

\section{Resumen}

El embarazo en adolescentes es una de las problemáticas más frecuentes en la población dominicana. Según el Fondo de Población de las Naciones Unidas (UNFPA), la República Dominicana está entre los cinco países de América Latina con mayor número de embarazos prematuros, con una tasa de natalidad en jóvenes entre 15 y 19 ańos del $20,5 \%$, en $2015^{1}$. Numerosos estudios han concluido que los factores sociodemográficos tienen una gran influencia en el embarazo precoz; debido a la falta de esfuerzos para realizar campañas nacionales de educación sexual, es preocupante la cantidad de adolescentes, principalmente entre 13 y 19 ańos, que quedan embarazadas, desconociendo los grandes riesgos que esto supone.

En este trabajo, analizamos el perfil sociodemográfico de las adolescentes embarazadas asistidas en una unidad de atención integral de un hospital de Santo Domingo, República Dominicana, durante el período febrero-abril de 2017, mediante un estudio prospectivo, descriptivo y de corte transversal, en el que se analizaron las características sociales implicadas en el embarazo temprano, a partir de una muestra de 130 adolescentes embarazadas. El estudio indicó que la edad más frecuente en la que se presenta el embarazo adolescente fue 19 años, con un $34.6 \%$. Del mismo modo, se determinó que

\footnotetext{
* Dr. Omar de la Rosa, ginecólogo-obstetra, endocrinólogo.

Hospital Dr. Reynaldo Almánzar, Santo Domingo, República

Dominicana. omardr2611@gmail.com

** Biointec
}

\section{Summary}

Adolescent pregnancy is one of the most frequent problems in the Dominican population. According to the United Nations Population Fund (UNFPA), the Dominican Republic is among the five countries in Latin America with the highest number of premature pregnancies, with a birth rate in young people between 15 and 19 years of $20.5 \%$, in 2015. Numerous studies have concluded that sociodemographic factors have a great influence on early pregnancy, and due to the lack of efforts in national sex education campaigns, the number of adolescents, mainly between 13 and 19 years old, who become pregnant is worrisome, ignoring the great risks that this entails.

We analyze the sociodemographic profile of pregnant adolescents assisted in a comprehensive care unit of a hospital in Santo Domingo, Dominican Republic, during the period February-April 2017, in a prospective, descriptive and cross-sectional study, in which analyzed the social characteristics in early pregnancy, from a sample of 130 pregnant adolescents. The study indicated that the most frequent age in which adolescent pregnancy occurs was 19 years, with $34.6 \%$. In the same way, it was determined that more than half of the samples were in a state of free union (53\%), equivalent 
más de la mitad de las muestras se encontraban en un estado de unión libre (53\%), equivalente a 69 casos; además, el sector más frecuente fue Villa Mella, con un 18.5\%; y más del $80 \%$ de las pacientes tenía seguro de salud público subsidiado por el gobierno.

Palabras clave: perfil sociodemográfico; adolescentes; embarazo; ginecología y obstetricia.

\section{Introducción}

El embarazo en adolescentes en la República Dominicana es un tema muy delicado que no recibe la importancia que merece. Cada día son más los casos de jóvenes menores de 19 años que resultan embarazadas, principalmente por la falta de conocimiento y educación en el ámbito sexual; incluyendo también la ausencia de métodos anticonceptivos ${ }^{2,3}$. Además, estudios previos como Determinantes del embarazo adolescente en Ecuador en el periodo 2011-2013, de Pérez Arrobo ${ }^{4}$, y Perfil sociodemográfico del embarazo y la maternidad adolescente, de Martes-Carmago ${ }^{5}$, indican que existe una gran relación entre el embarazo precoz y los factores sociodemográficos de las adolescentes.

El objetivo de esta investigación fue analizar el perfil sociodemográfico de las adolescentes embarazadas y el grado de influencia que tienen estas características sociales. La importancia del trabajo radica en que a partir de dar a conocer estos datos puede incrementarse consecutivamente la posibilidad de reducir la cantidad de embarazos en adolescentes, de manera que se puedan evitar las graves consecuencias que esto conlleva. Del mismo modo, se espera que los servicios de salud enfaticen en brindar una educación sexual adecuada, tarea imprescindible para la prevención del embarazo precoz.

Se denomina embarazo en adolescentes al que ocurre durante la adolescencia de la madre, definida esta última por la Organización Mundial de la Salud (OMS) como el lapso de vida transcurrido entre los 10 y los 19 años de edad. También se suele designar to 69 cases; in addition, the most frequent sector was Villa Mella, with $18.5 \%$, and more than $80 \%$ of the patients had public health insurance subsidized by the government.

Keywords: Sociodemographic profile; adolescents; pregnancy; gynecology and obstetrics.

como embarazo precoz, en atención al que se presenta antes de que la madre haya alcanzado la suficiente madurez emocional para asumir la compleja tarea de la maternidad ${ }^{6}$.

El embarazo en adolescentes generalmente viene acompañado de grandes complicaciones en la salud de la madre, tales como anemia grave, amenaza de aborto, parto prematuro, hipertensión inducida por el propio embarazo, placenta previa e infecciones, entre otras. En la salud del niño puede presentarse desde bajo peso al nacer hasta secuelas que en ocasiones son para toda la vida, como retraso mental y desarrollo biológico deficiente, etc ${ }^{7}$.

El embarazo en la adolescencia se relaciona con factores que incluyen: nivel socioeconómico bajo, lugar donde viven, nivel de educación, inestabilidad familiar, iniciación temprana de la actividad sexual, falta de conocimientos sobre educación sexual e irresponsabilidad por parte de los adolescentes. Además, se puede evidenciar que los embarazos sucesivos en adolescentes contribuyen a perpetuar el ciclo de la pobreza ${ }^{8}$. La fecundidad adolescente es mayor en países en vías de desarrollo y entre las clases sociales menos favorecidas. La disminución de la edad de la menarquia, la demora para casarse, la contracepción inadecuada y la pobreza han influido en el aumento del embarazo en adolescentes?

Una investigación realizada en 16 países de América Latina concluyó que el ser hijo o hija de madre adolescente perteneciente a un hogar pobre reduce la posibilidad de que estos culminen la enseñanza secundaria y 
que se pueda romper la transmisión de la pobreza de generación en generación ${ }^{10}$. Al no terminar la escuela, la educación que estas tienen es deficiente, aumentando la probabilidad de tener un embarazo antes de tiempo. Se demostró que las adolescentes empobrecidas y con una educación deficiente son más proclives a quedar embarazadas que las adolescentes con más recursos económicos y una mejor formación?.

En un hospital de Santo Domingo, la Maternidad La Altagracia, el 32\% de los partos anuales pertenece a las adolescentes, y el $85 \%$ de estas jóvenes madres vive con su pareja fuera de su casa mater$\mathrm{na}^{11}$. Muchos de los adolescentes sexualmente activos están casados o tienen una pareja estable, otros están solteros. En otras palabras, existen varios grupos de adolescentes con necesidades diversas. A esto hay que sumar el ámbito heterogéneo en que puede desenvolverse cada uno en el plano social, psicológico, emocional, étnico y cultural ${ }^{12}$.

\section{Material y métodos}

Esta investigación fue de carácter prospectivo, debido a que fue realizada durante el período febrero-abril de 2017 y sus datos fueron recolectados de fuentes secundarias; descriptivo, pues se detallaron los resultados tal como se presentaron; de tipo transversal, ya que se hizo un corte en el tiempo, midiendo las variables solo en una ocasión, sin dar continuidad y/o seguimiento a estas en el eje cronológico. Los datos fueron recolectados mediante la revisión de registros médicos pertenecientes a las adolescentes embarazadas que asistieron a una unidad de atención integral de un hospital de Santo Domingo, República Dominicana, obteniendo resultados que reflejaron la inferencia del perfil sociodemográfico en el embarazo precoz. Las variables estudiadas incluyeron la edad de las adolescentes embarazadas, el estado civil, así como la posición socioeconómica y geográfica.

La muestra del estudio fue de 130 pacientes, tomadas al azar, estableciendo como criterio de inclusión que fueran adolescentes embarazadas, es decir, entre las edades de 10 y 19 años. Antes de la aplicación de este protocolo, se procedió al consentimiento informado con las pacientes y se aseguró a las encuestadas la absoluta confidencialidad de la información. Los datos obtenidos fueron analizados estadísticamente mediante el programa Microsoft Excel, versión Office 2016.

\section{Resultados}

En la gráfica 1 se observa la distribución de las adolescentes embarazadas según su edad.

\section{Gráfica 1: Distribución de adolescentes embara- zadas según edad (n=130)}

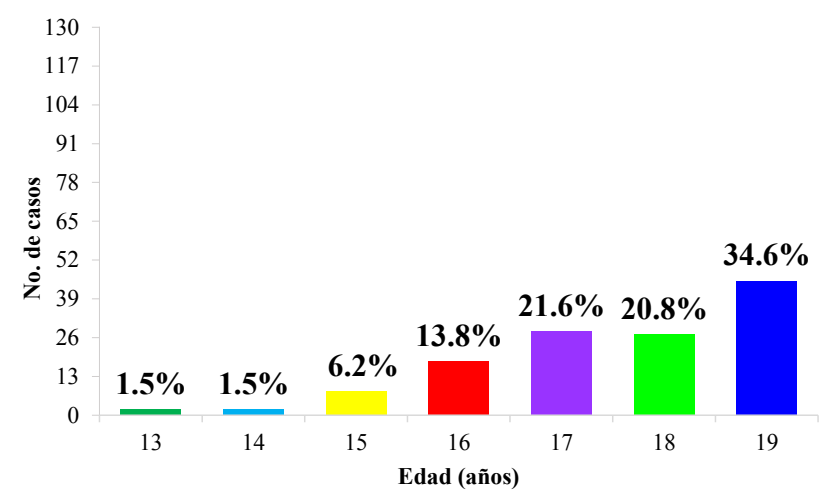

De acuerdo a los resultados obtenidos, se observa que la mayoría de las adolescentes embarazadas tenía 19 años de edad, con un 34.6\%, correspondiente a 45 sujetos de la muestra. A continuación, le siguen las adolescentes que tenían 17 años de edad, con un $21.6 \%$, que representa 28 pacientes; un $20.8 \%$ de las adolescentes tenía 18 años, equivalente a 27 adolescentes; un 13.8\%, perteneciente a adolescentes con 16 años, con un total de 18 sujetos; un $6.2 \%$, que corresponde a adolescentes con 15 años, representando a 8 pacientes; y un 1.5\% para las adolescentes con 14 años, con un total de 2 adolescentes, igualando el $1.5 \%$ de las adolescentes con 13 años embarazadas.

La gráfica 2 muestra el estado civil de las adolescentes embarazadas. 
Gráfica 2: Estado civil de las adolescentes embarazadas $(n=130)$

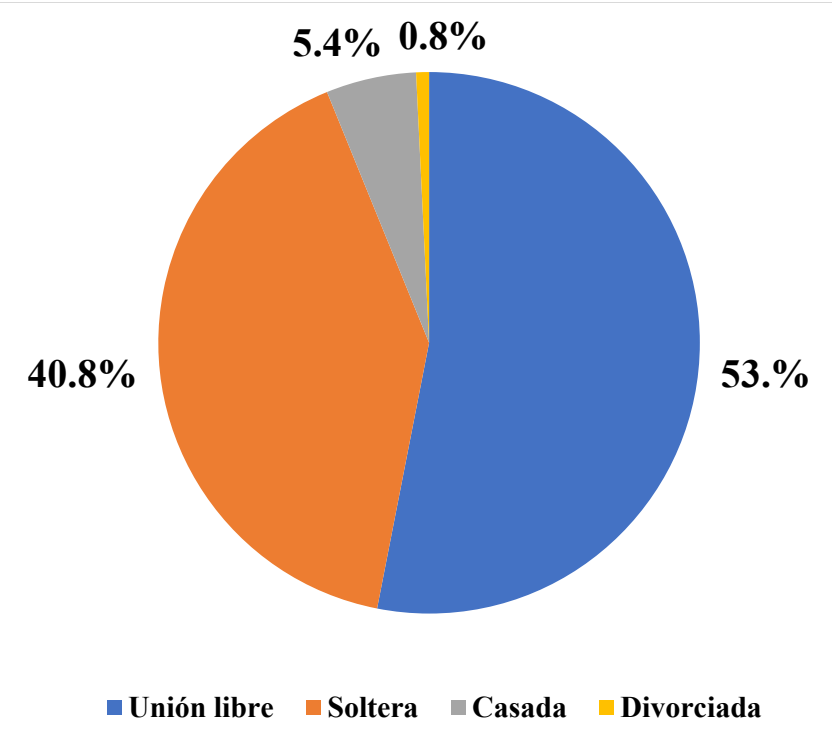

En la gráfica anterior se puede apreciar que un 53\% de las adolescentes embarazadas vivía en unión libre, correspondiente a 69 sujetos de la muestra. Mientras que un $40.8 \%$ estaban solteras, representando 53 de las adolescentes de la muestra; el 5.4\% de las adolescentes embarazadas estaban casadas, equivalente a 7 pacientes; y, por último, el $0.8 \%$ estaban divorciadas, con un total de 1 sujeto de la muestra.

A continuación, en la tabla 1 se presenta la distribución de las adolescentes embarazadas según su posición geográfica.

Según los resultados obtenidos, en la gráfica se muestra que el sector donde vivía la mayor cantidad de adolescentes embarazadas fue Villa Mella, con 24 casos, correspondientes al $18.5 \%$ de la muestra total. Le sigue el sector de Los Guaricanos, donde vivían un total de 17 adolescentes embarazadas, equivalentes al 13.1\%; a continuación, el sector de Sabana Perdida ocupa el $10 \%$, con un total de 13 sujetos de la muestra inicial.

La gráfica 3 permite apreciar el tipo de seguro de salud que usaron las adolescentes que formaron parte del estudio.

\section{Gráfica 3: Tipo de seguro de salud utilizado por las adolescentes embarazadas $(n=130)$}

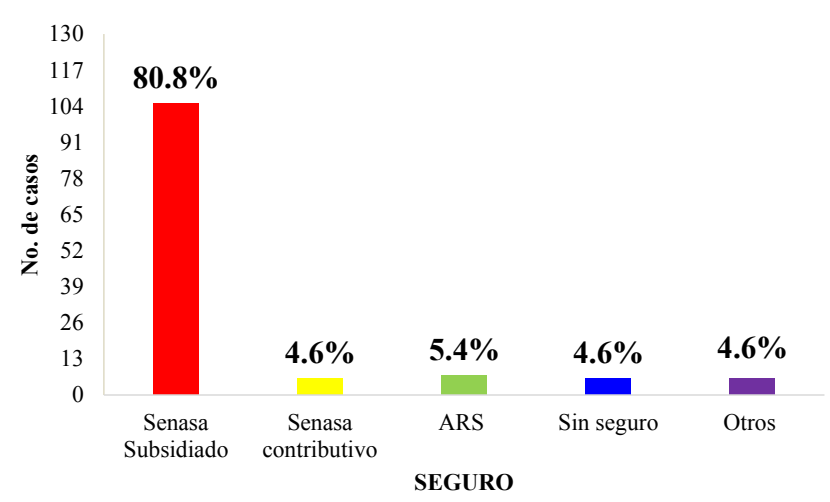

En la gráfica se observa que el seguro más utilizado por las adolescentes embarazadas fue el Senasa subsidiado, con un $80.8 \%$, correspondiente a 105 de las adolescentes de la muestra. A continuación, le sigue el ARS, con un $5.4 \%$, equivalente a 7 sujetos de la muestra; un total de 6 pacientes usaba el Senasa contributivo, representando el 4.6\%; 6 de las pacientes estudiadas no tenían seguro, correspondiente a un $4.6 \%$ e igualando a la cantidad de adolescentes que usaban otros seguros de minoría.

\section{Discusión}

La edad más frecuente de las adolescentes embarazadas en esta investigación fue de 19 años, con un $34.6 \%$. Según un estudio publicado en 2012, titulado Embarazo adolescente y oportunidades en América Latina y el Caribe: sobre maternidad temprana, pobreza y logros económicos, de los autores Azevedo et al. ${ }^{13}$, la edad más común de las adolescentes embarazadas oscila entre 15 y 19 años, lo que coincide con los resultados de la investigación.

Del $100 \%$ de la muestra estudiada, los resultados mostraron que más de la mitad de las adolescentes embarazadas se encontraba en un estado de unión libre (53\%), correspondiente a 69 casos. Más del $40 \%$ de ellas (53 casos) se encontraban solteras; 7 adolescentes estaban casadas, para un $5.4 \%$, y el $0.8 \%$ restante correspondió a una adolescente 


\section{Tabla 1: Distribución de la posición geográfica de las adolescentes embaraza- das $(\mathbf{n}=130)$}

\begin{tabular}{lclc}
\hline Sectores & No. de casos & Sectores & No. de casos \\
\hline 2 de enero & 1 & Las Cañitas & 2 \\
Arroyo Hondo & 2 & Las Palmas & 1 \\
Barrio Agrario & 1 & Los Coquitos & 1 \\
Boca Chica & 3 & Los Frailes & 2 \\
Brisas del Este & 6 & Los Guandules & 1 \\
Cancino Adentro & 8 & Los Guaricanos & 17 \\
Capotillo & 1 & Los Mina & 5 \\
Centro de la Ciudad & 1 & Los Tres Brazos & 1 \\
Charles de Gaulle & 1 & Mal Nombre & 1 \\
Cristo Rey & 2 & Mata San Juan & 1 \\
Cruz Grande & 1 & Mendoza & 1 \\
El Almirante & 2 & Pedro Brand & 2 \\
Guerra & 3 & Sabana Perdida & 13 \\
Hacienda Estrella & 1 & San Luis & 2 \\
Hainamosa & 3 & Santa Cruz & 1 \\
Invivienda & 1 & Villa Faro & 1 \\
KM 10 1/2 & 1 & Villa Hermosa & 1 \\
La Ceiba & 1 & Villa Mella & 24 \\
La Gina & 1 & Villas Agrícolas & 1 \\
La Victoria & 7 & Yamasa & 3 \\
La Zursa & 2 & & 130 \\
\hline
\end{tabular}

Fuente: Unidad Atención Integral Hospital Materno Infantil Dr Reynaldo Almanzar

que estaba divorciada. De acuerdo con un estudio realizado en el año 2012, titulado Embarazo en adolescentes y sus repercusiones materno perinatales, de los autores Panduro et al..$^{14}$, la mayoría de las adolescentes vivía en unión libre (62.7\%), seguido de las que se encontraban solteras $(28.7 \%)$, casadas $(8.5 \%)$ y divorciadas $(0.1 \%)$, lo que concuerda con los resultados obtenidos en la investigación ${ }^{15}$. Estos resultados mostraron que una gran cantidad de adolescentes se encontraban solteras durante el embarazo, lo que puso en evidencia la irresponsabilidad por parte de los jóvenes al momento de tener relaciones sexuales.
Villa Mella fue el sector donde se hallaron más adolescentes embarazadas (18.5\%), seguido de otros sectores como Los Guaricanos (13.08\%) y Sabana Perdida (10\%). Estos sectores tienen un bajo nivel socioeconómico y educativo, lo que permite inferir que la cantidad de adolescentes embarazadas está estrechamente relacionada con su localización geográfica, relacionándolo, a su vez, con el nivel económico y educativo propios de estos sectores. La investigación Diagnóstico de la situación del embarazo en la adolescencia en Chile, 2008, del Ministerio de Salud, Gobierno de Chile, UNFPA y FLASCO-Chile ${ }^{15}$, reveló que 
el $65 \%$ de los nacimientos de hijos/as de madres adolescentes ocurre en zonas rurales, donde hay menos recursos económicos; mientras que un 35\% pertenece a las zonas urbanas, donde existe mayor nivel económico. Este estudio llegó a la conclusión de que existe un vínculo entre la tasa del embarazo adolescente y el sector donde estas residen, corroborando los datos reflejados en la presente investigación.

Según otro estudio, que lleva por título Madres adolescentes en República Dominicana, de la Oficina Nacional de Estadística (ONE), las condiciones socioeconómicas influyen en la fecundidad adolescente. El tipo de seguro más utilizado fue el Senasa subsidiado, con más de un $80 \%$, lo que significa que la gran mayoría de las pacientes asistidas en este hospital era de bajos recursos, porque este es un servicio cubierto totalmente por el Estado. Mientras que el $19.2 \%$ de las pacientes pertenecía a una posición socioeconómica más elevada, debido a que utilizan seguro de salud privado, lo que confirma lo dicho en el estudio previamente mencionado, que el nivel socioeconómico de las pacientes interviene en gran medida en la tasa de embarazos ${ }^{16}$.

Luego de haber presentado los resultados obtenidos en la investigación, se concluye que los factores sociodemográficos tienen una gran influencia en el embarazo adolescente, siendo la edad, el estado civil, la posición geográfica y la posición socioeconómica algunos de los factores más relevantes.

\section{Bibliografía}

1. EFE. Tasa de embarazo adolescente en República Dominicana es de 20,5 \%, quinta en Latinoamérica. Acento, República Dominicana: 1 ro de marzo de 2017. Sección Actualidad.1 p.

2. Panday S, Makiwane M, Ronchad C, Letsoalo T. Teenage pregnancy in South Africa: With a specific focus on school-going learners. Child, Youth, Family and Social Development, Human Sciences Research Council. Pretoria, África: Departamento de educación básica; 2009. Unicef $53 \mathrm{p}$.

3. Leyton C, Bardi L, González E, Molina T, Oneto C. Uso del preservativo en adolescentes nuligestas con uso de anticonceptivos hormonales. Rev SOGIA [Internet]. 2006;13(1):1624. Disponible en: http://www.cemera.cl/ sogia/pdf/2006/SOGIA_1_2006_03_.pdf

4. Pérez Arrobo G. Determinantes del embarazo adolescente en Ecuador en el período 20112013 [tesis doctoral]. Ecuador: Pontificia Universidad Católica del Ecuador Facultad de Economía; 2016.

5. Martes-Carmago P. Perfil sociodemográfico del embarazo y la maternidad adolescente. Análisis del período 1986-2014. 1 1 ed. Perú: Aires de Crear S.A.C.; 2016. 53 p.

6. Beltrán Molina L. Embarazo en adolescentes. Caracas: Fundación Escuela de Gerencia Social; 2006.

7. Blázquez Morales MS. Embarazo adolescente. REMSyS. 2012; 3(1): 2.

8. Montalvo González N. Factores que predisponen al embarazo en adolescentes [tesis doctoral]. Veracruz, México: Universidad Veracruzana; 2007.

9. Vallejo Barón J. Embarazo en adolescentes: complicaciones. RMCC [Internet] 2013 [citado 25 abril 2017]; 70(605): 65-69. Disponible en: http://www.medigraphic.com/pdfs/revmedcoscen/rmc-2013/rmc131m.pdf

10. Aldaz-Carolli E, Morán R. Escaping the poverty trap in Latin America: The role of family factors. Cuadernos de economía Año 38, No.114, pp.155-190. 2001 [Internet]. 
[citado 26 abril 2017]; 114(38). Disponible en: http://www.scielo.cl/scielo.php?script=sci_arttext\&pid=S071768212001011400003

11. Mejía M. El 85\% menores paren Maternidad La Altagracia viven con su pareja. Diario Libre, República Dominicana: 3 de junio de 2009. Noticias pág. 12.

12. Azevedo JP, Favara M, Haddock SE, LópezCalva LF, Müller M, Perova E. Embarazo adolescente $y$ oportunidades en América Latina y el Caribe: sobre maternidad temprana, pobreza y logros económicos. Washington DC, Estados Unidos: Banco Internacional de Reconstrucción y Fomento/Banco Mundial; 2012. Disponible en: http://documents.worldbank.org/curated/en/983641468238477531/ pdf/831670WPOSPANI0Box0382076B00PUBLIC0.pdf

13. Azevedo JP, Favara M, Haddock SE, LópezCalva JL, Muller M, Perova M. Embarazo adolescentes y oportunidades en América
Latina y el Caribe: sobre maternidad temprana, pobreza y logros económicos. Washington, Estados Unidos: Banco Mundial; 2012.

14. Panduro J, Jiménez P, Pérez J, Panduro E, Peranza D, y Quezada N. Embarazo en adolescentes y sus repercusiones materno perinatales. Ginecol Obs Mex [en línea]. 2012 [citado 26 abril 2017]; 80(11):694-704. Disponible en: http://www.medigraphic.com/pdfs/ginobsmex/gom-2012/gom 1211c.pdf

15. Ministerio de Salud, Gobierno de Chile, UNFPA CHI1R11A y FLASCOChile. Diagnóstico de la situación del embarazo en la adolescencia en Chile 2008. $1^{\text {a }}$ ed. Santiago de Chile: Alfabeta Artes Gráficas; $2009.58 \mathrm{p}$.

16. Oficina Nacional de Estadística. Madres adolescentes en República Dominicana. Santo Domingo, República Dominicana: Oficina Nacional de Estadística; 2008. 31 p. 